\title{
Thick-Film Tilt Sensors: Feasibility Study Example Using Free Convective Motion of An Heating Air Mass
}

\author{
D. Crescini, and M.Romani \\ Dipartimento di Ingegneria dell'Informazione \\ Facoltà di Ingegneria - Università di Brescia - Via Branze 38 - 25123 Brescia - Italy \\ Tel. +390303715547 - Fax +39030380014 \\ E_mail: damiano.crescini@ing.unibs.it
}

\begin{abstract}
In this application a feasibility study example of thick-film tilt sensor has been presented based on heat transfer by natural convection. The device measures internal changes in heat transfer due to the inclination. The device is functionally equivalent to traditional proof-mass accelerometer. The proof mass in the new thick-film sensor is a gas. The gaseous proof-mass provides great advantages over the use of the traditional solid proof mass. A conditioning electronics amplifies the signal variation induced by the inclination compensating the deviation due to the initial asymmetrical values of the bridge arms and their slow variations. Preliminary tests on the first prototypes show an accuracy of about $2 \%$ full scale output, repeatability of about $0.2^{\circ}$ and resolution better than $0.1^{\circ}$ over $\mathrm{a} \pm 20^{\circ}$ range.
\end{abstract}

\section{Introduction}

Many vehicles require the use of inclinometers to control their stability to prevent rolling over or flipping due to improper handling, especially in off-road environment. Moreover inclination sensors with reduced power supply and minor cost/performances ratio expand their use in applications such as security, computer peripherals and so on. The use of screen-printing thick-film technology in the field of sensors started about 20 years ago [1],[2],[3],[4]. The advantages of this technology in electronics are also apply to the sensor field, as for example:

- low investment for equipment

- versatility in the sensor design (size of the substrate, size, shape and nature of the metallic contacts, of the heating elements etc.)

- mass production of customized sensors at low cost

Should be noted however, that the technology is also widely used for the fabrication of chemical and mechanical sensors and many commercial devices exist $[5,6]$. The integrability of the technology also allows combination with other enabling technologies, such as silicon, to provide powerful and economically viable solid-state sensors.

\section{The operating principles and the fabrication method in thick-film technology}

The principle of operation of the TFT inclinometer is based on heat transfer by natural convection. The device measures internal changes in heat transfer due to the inclination caused by the gravity force. The device is functionally equivalent to traditional proof-mass accelerometers. The proof mass in the new thickfilm sensor is a gas. The gaseous proof-mass provides great advantages over the use of the traditional solid proof mass. The device does not show striction and particle contamination problems associated with competitive devices and provides a high shock survival leading to significantly lower failure rates and lower loss due to handling during assembly. The arrangement necessary for measuring the inclination effects on heat transfer is described next. A double structure heat source, centered in the ceramic substrate is suspended across a cavity. Equally spaced temperature sensors are located equidistantly on all two sides of the heat source. Under zero inclination, a temperature gradient is symmetrical to the heat source, so that the temperature is the same at all two temperature sensors, causing them to output the same voltage (reference Figure $1(\mathrm{~A})$ ). Inclination in the sensing direction will disturb the temperature profile, due to free convection heat transfer, causing it to be asymmetrical (reference Figure 1 (B)). The temperature, and hence voltage 


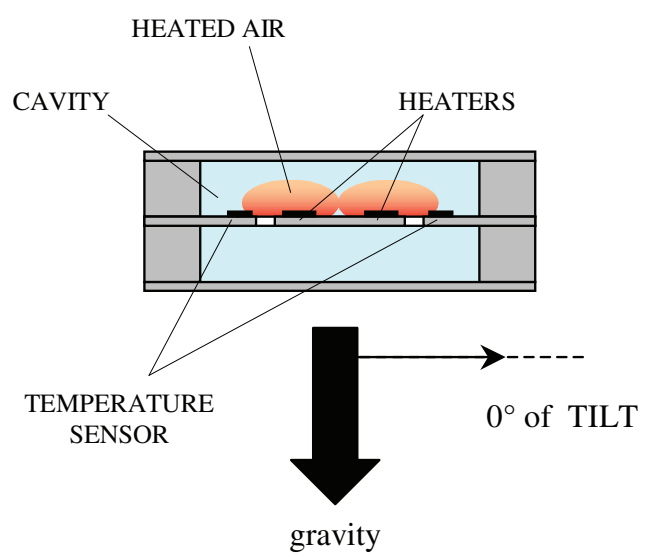

Symmetrical Temperature Profile

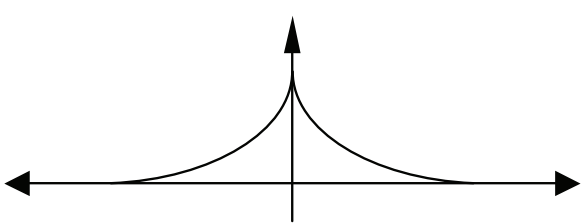

(A)

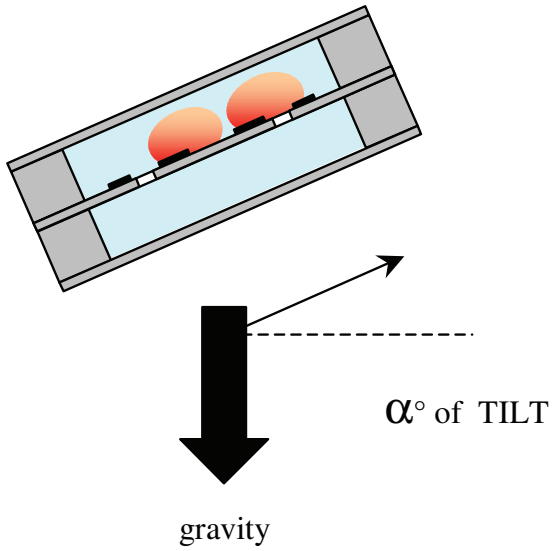

Asymmetrical Temperature Profile

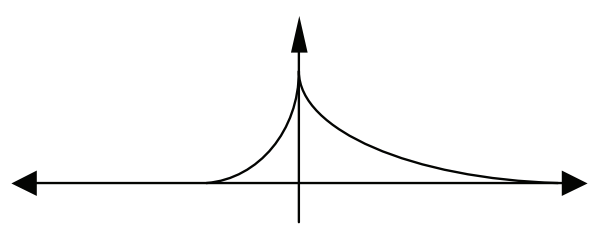

(B)

Fig.1. Vertical cross-section showing the sensing sequence (a) $0^{\circ}$ TILT angle (b) $\alpha^{\circ}$ TILT angle.

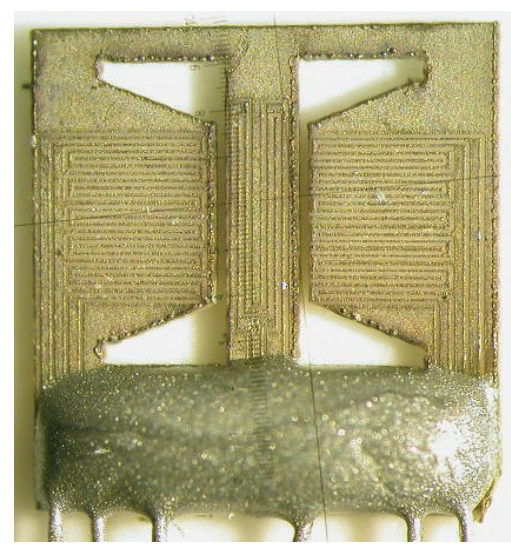

Fig. 2. Photograph of the TFT substrate.

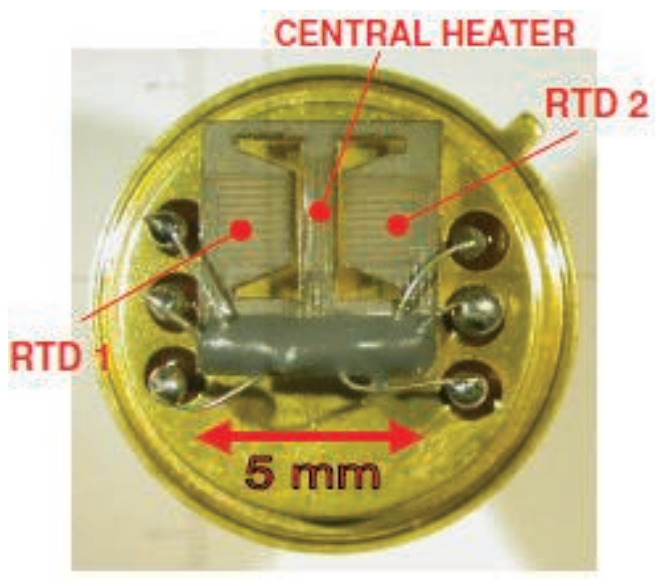

Fig. 3 - Photograph of the tilt sensor prototype in thick-film technology

output of the two temperature sensors will then be different. The differential voltage at the temperature sensor outputs is directly proportional to the inclination. The TFT tilt sensor is most sensitive to changes in angular position, or tilt, when the sensitive axis is perpendicular to the force of gravity, or parallel to the Earth's surface. Similarly, when the sensitive axis is parallel to the force of gravity (perpendicular to the Earth's surface), it is least sensitive to changes in tilt. The fabrication method involves the standard thickfilm procedures on $96 \%$ alumina substrate. The planar resistors adopted for the heating processes and the thermal sensors are based on a Pt/Au ink. In order to achieve adequate electrical characteristics, a firing process has been adopted at a peak temperature of about $950^{\circ} \mathrm{C}$. After the screen-printing and firing processes the ceramic substrate, covered by the conductive layer (thickness of about $25 \mu \mathrm{m}$ ) is cut by laser to obtain central heater's support and the double bridge structure for the thermal sensors. The final configuration of the heater and the thermoresistors are then obtained by laser scribing. In Figure 2 a photograph of the TFT sensor substrate is reported. The central vertical bar hosts the resistance track of the heater while the two vertical lines equidistant and close to the bar are the two termoresistances

Figure 3 shows a photograph of the TFT inclinometer sensor into a TO package. The dimension of the sensor is $4 \mathrm{~mm} \mathrm{X} 6 \mathrm{~mm}$. 


\section{Signal conditioning electronics}

A block scheme of the conditioning electronics is reported in Figure 4. $\mathrm{H}$ is the heater resistance driven by a generator whose current is modulated by an $\alpha(t)$ factor equal to $A \sin \left(2 \pi f_{c} t\right) . T_{1}$ and $T_{2}$ are the two thermoresistances: both have one common terminal linked to the input of a trans resistance amplifier. The other two terminals of $\mathrm{T}_{1}$ and $\mathrm{T}_{2}$ are respectively tied to a positive voltage reference and to the output of an integrator. The amplifier, the integrator and $\mathrm{T}_{2}$ are a negative feedback system whose function is to compensate slow frequency variations. The output of the feedback system is multiplied by $\alpha(\mathrm{t})$ and low pass filtered. These last two blocks function as a synchronous demodulator and the output $\mathrm{V}_{\mathrm{O}}$ is the sensor output. Into the common node, $\mathrm{T}_{1}$ and $\mathrm{T}_{2}$ currents are subtracted. The positive integrator regulates its output voltage until the low-frequency current flowing into $\mathrm{T}_{2}$ is equal and opposite to the lo- frequency current of $\mathrm{T}_{1}$, zeroing, in this way, the voltage output of the trans resistance amplifier. The detailed scheme of the feedback system is shown in Figure 5: R1 e R2 represent the resistances of the thermoresistances $\mathrm{T}_{1}$ and $\mathrm{T}_{2}, \mathrm{R} 3$ and the A1 amplifier constitutes the negative trans resistance amplifier, and the other four elements (marked as R and C) together with A2 constitute the positive integrator. The transfer function between the current $\Delta \mathrm{I}$ injected into the negative terminal of $\mathrm{A} 1$ amplifier and the $\mathrm{V}_{1}$ is:

$$
\frac{V_{1}}{\Delta I}=-\frac{s C R R_{2} R_{3}}{s C R R_{2}+R_{3}}
$$

corresponding to an high pass filter having a cut off frequency of :

$$
f_{t}=\frac{R_{3}}{2 \pi C R R_{2}}
$$

Since $\mathrm{R} 1 \cong 50 \Omega, \mathrm{R} 2 \cong 50 \Omega, \mathrm{R} 3=10 \mathrm{k} \Omega, \mathrm{R}=3.3 \mathrm{M} \Omega$ and $\mathrm{C}=10 \mu \mathrm{F}, \mathrm{f}_{\mathrm{t}}$ is equal to $1 \mathrm{~Hz}$. In this case slow drift and initial mismatching of the two thermoresistances are compensated and do not influence the sensor output. Because $\alpha(\mathrm{t})$ is a sinusoid with a frequency $f_{c}(30 \mathrm{~Hz})$ greater than $f_{t}$ the cut-off frequency, the heating mass induces a $\Delta R 1$ and $\Delta R 2$ changes, modulated again at $\mathrm{f}_{c}$, into $\mathrm{R} 1$ and $\mathrm{R} 2$ respectively. Choosing $\mathrm{f}_{\mathrm{c}}$ greater than $\mathrm{f}_{\mathrm{t}}$, the difference between $\Delta \mathrm{R} 1$ and $\Delta \mathrm{R} 2$ comes directly to the syncrounous demodulator. When the sensor is parallel to the horizontal line, $\Delta \mathrm{R} 1$ is equal to $\Delta \mathrm{R} 2$ and because $\mathrm{R} 1$ and $\mathrm{R} 2$ are driven by voltages of opposite signs, the current induced, namely $\Delta \mathrm{I} 1$ and $\Delta \mathrm{I} 2$, are equal and opposite producing a zero output voltage Vo. Applying an inclination to the sensor, due to the asymmetric profile of temperature, $\Delta \mathrm{R} 1$ and $\Delta \mathrm{R} 2$ have opposite signs and their corresponding currents $\Delta \mathrm{I} 1$ and $\Delta \mathrm{I} 2$ have same signs; they are summed and amplified of a $-10^{4}$ factor and through the synchronous demodulator generates a signal $\left(\mathrm{V}_{\mathrm{O}}\right)$ proportional to the inclination.

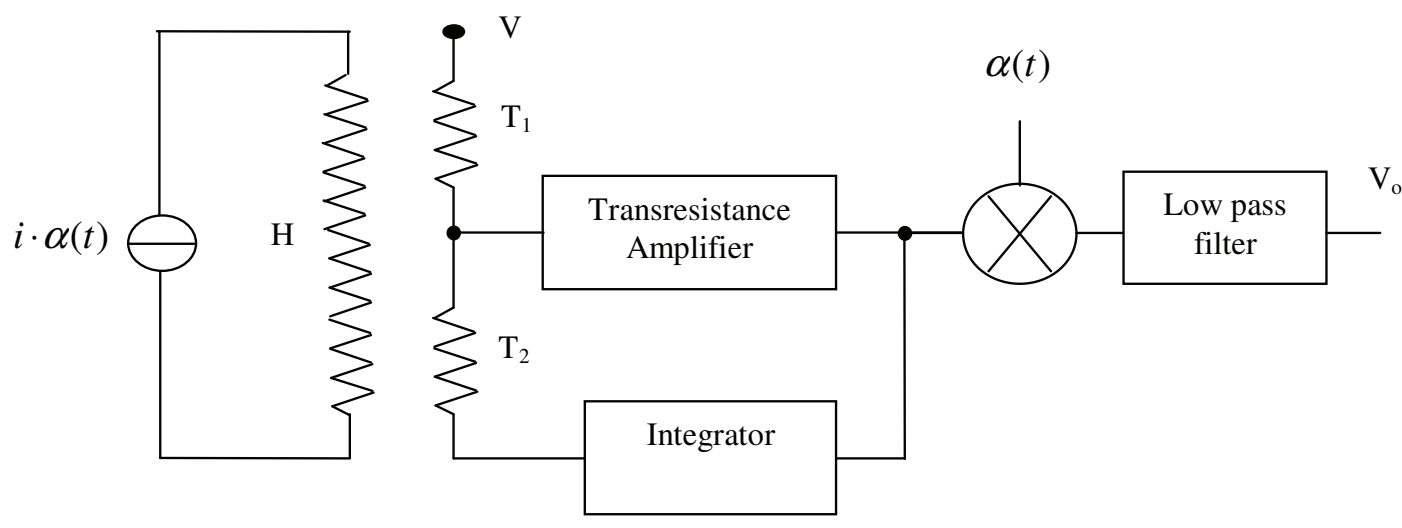

Fig. 4. Block scheme of the conditioning electronics, $\mathrm{T}_{1}$ e $\mathrm{T}_{2}$ are the two thermoresistances while $\mathrm{H}$ is the heater. 


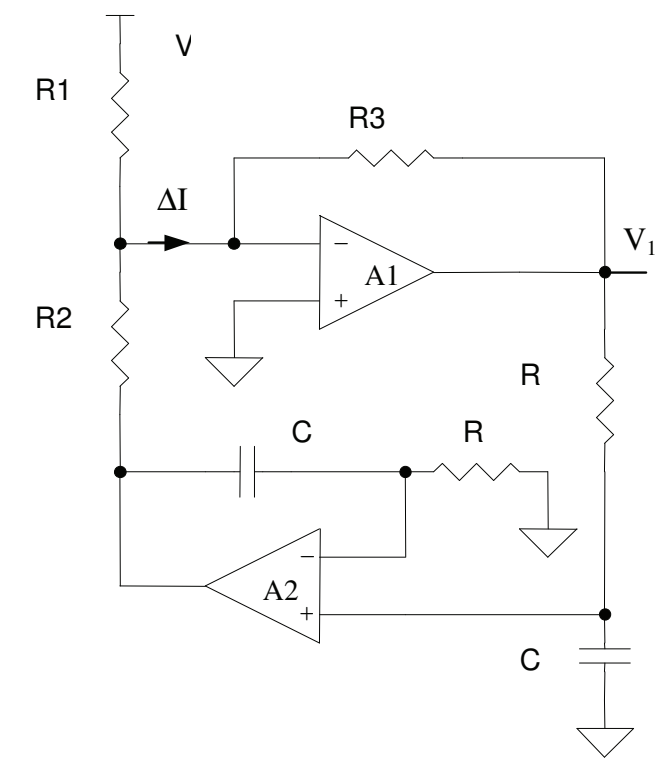

Fig. 5. Circuit details of the transresistance and integrator blocks.

\section{Experimental results}

Preliminary tests on the first prototypes show a sensitivity of about $8 \mathrm{mV} /{ }^{\circ}$ from $+50^{\circ}$ to 0 . Figure 6 illustrates, in the case of a constant room temperature, the inclinometer output at different inclination angles. Figure 7 reports, the percentage deviation of the experimental data from the regression line.

A maximum error of about $3^{\circ}$ can be found in the case of $\pm 50^{\circ}$ full scale output. Differently, a lower nonlinearity errors The repeatability has been evaluated and a value less than $0.2^{\circ}$ has been observed. Thermal tests will be performed in the near future. The total supply current is less than $20 \mathrm{~mA}$.

Note that the voltage output change for a small inclination angle is relatively small $\left(8 \mathrm{mV}\right.$ for $\left.1^{\circ}\right)$, so careful consideration to noise must be applied in the TFT inclinometer design. Reducing the low pass filter of the synchronous demodulator to $1 \mathrm{~Hz}$, a noise level in the order of $0.1 \mathrm{mVrms}$ has been measured. With such value a resolution better than $0.1^{\circ}$ should be obtained.

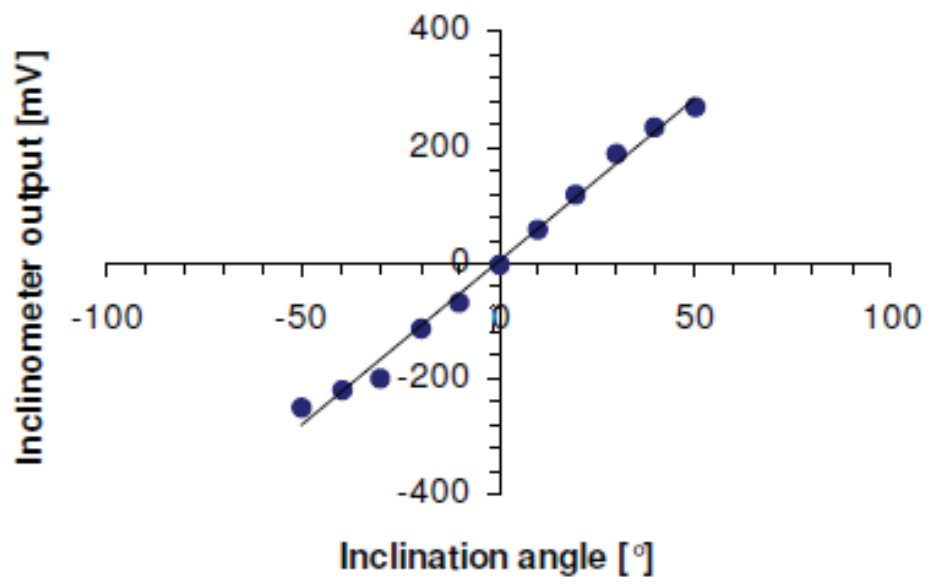

Fig. 6. Gravity component vs. Inclination angle. 


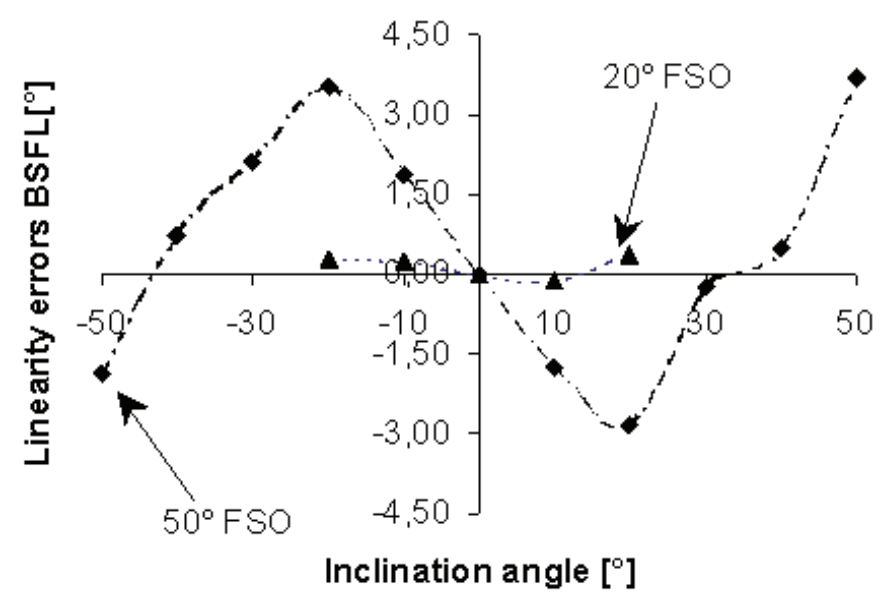

Fig. 7. Gravity component vs. Inclination angle.

\section{Conclusions}

Using thick-film technology on a ceramic substrate, a new tilt sensor based on the heat transfer principle has been developed. The sensor is fabricated from ceramic materials and thick-film technology. From the preliminary test the accuracy has been evaluated of about $2 \%$ full scale output while the repeatability of about $0.2^{\circ}$ over a $\pm 20^{\circ}$ range. The TFT tilt sensor is capable of resolving less $0.1^{\circ}$. Due to the compensation of the conditioning electronics, no trimming operation on the thick film resistors is necessary, contributing to the reduction of the overall fabrication costs.Finally, the sensor is inexpensive enough to be, in the near future, competitive with other types of commercial tilt transducer.

\section{References}

[1] M. Prudenziati and B. Morten, "The State of the Art in thick-film Sensors", Microelectronics Journal, n 23, pp.133-141 (1992)

[2] Brignell, J. E., White, N. M. and Cranny, A. W. J.:'Sensor applications of thick-film technology', IEE Proc. Pt. I, 1988, 135 , No. 4, pp 77-84.

[3] D. Crescini, D. Marioli, E. Sardini and A.Taroni "Large bandwith and thermal compensated piezoelectric thick-film accelerometer transducer", Sensor and Transducer A 87 (2001) pp. 131-138

[4] D. Crescini, D. Marioli, V. Ferrari, and A.Taroni "Vibration and vibrating sensor in thick-film technology", Machine Vibration 4 (1995) pp.161167

[5] TFT pressure Sensors proposed by GEFRAN SENSORI SPA - Italy

[6] TFT chemical Sensors proposed by Metallux Electronic SA - Switzerland 